\title{
Periradicular corticosteroid infiltration for radicular pain - comparison of Diprophos and Depomedrone and ozone effects
}

\author{
David Krahulik', Miroslav Vaverka', Lumir Hrabalek', Daniel Pohlodek', Jakub Jablonsky', Jan Valosek¹,2 , Jana Zapletalova ${ }^{3}$
}

Objectives. To determine the treatment effect of corticosteroids in periradicular therapy (PRT) for radicular pain and to compare different types of corticosteroids and ozone. We also examined the effect in different indication groups for periradicular therapy for each type of treatment agent.

Background. Various studies have examined the therapeutic value of periradicular infiltration using treatment agents consisting of local anesthetic and corticosteroids or ozone application for radicular pain. This is the first study to compare different types of corticosteroids and ozone.

Methods. Eligible patients with radicular pain who failed conservative management were divided into five indication groups and prospectively followed to assess the PRT effect of corticosteroids or ozone application. PRT was performed under computer tomography (CT) monitoring. A set of three PRT applications in three weeks was applied and the outcome was evaluated using a visual analogue score for back and leg pain. The in-group and between-group treatment effect was tested using the Wilcoxon signed-rank test and the Kruskal-Wallis H-test with Dunn's post-hoc tests, respectively. The dependency between treatment effectiveness and indication for each group was tested using the Kruskal-Wallis H-test and Dunn's post-hoc tests.

Results. We prospectively followed 150 patients, randomized into three groups of 50 patients each. The follow-up rate was $100 \%$. All three treatment agents showed a statistically significant treatment effect $(P<0.001)$. The statistically significant effect was higher in betamethasone (Diprophos) versus methylprednisolone (Depomedrone) $(P=0.019)$ and Diprophos versus ozone $(P<0.001)$. Diprophos also showed the highest decrease of VAS after therapy versus VAS prior to therapy (median decrease $=4$ ) compared to Depomedrone and ozone (median decrease $=3$ and 2, respectively). The statistically significant outcome was better with the indication of spondylolisthesis and disc herniation $(P=0.019)$ indication for the Diprophos group and between spinal stenosis and spondylolisthesis $(P=0.022)$ and spondylolisthesis and disc herniation $(P=0.016)$ for the ozone group.

Conclusion. Clinical improvement occurred in all three groups but Diprophos showed the statistically best treatment effect compared to Depomedrone and ozone. Disc herniation resulting in radicular pain had a statistically significant better effect in comparison with spondylolisthesis in the Diprophos and ozone groups, but the ozone group showed heterogeneity depending on treatment effect and indication.

Key words: computer tomography, corticosteroids, radicular pain, ozone

Received: June 29, 2021; Revised: October 26, 2021; Accepted: October 26, 2021; Available online: November 15, 2021 https://doi.org/10.5507/bp.2021.061

(c) 2023 The Authors; https://creativecommons.org/licenses/by/4.0/

'Department of Neurosurgery, Faculty of Medicine and Dentistry, Palacky University Olomouc and University Hospital Olomouc, Czech Republic

${ }^{2}$ Department of Neurology, Faculty of Medicine and Dentistry, Palacky University Olomouc and University Hospital Olomouc, Czech Republic ${ }^{3}$ Department of Biophysics, Faculty of Medicine and Dentistry, Palacky University Olomouc, Czech Republic Corresponding author: David Krahulik, e-mail: david.krahulik@fnol.cz.

\section{INDRODUCTION}

Sciatic pain is a one of the most common causes of disability in the adult population. It can be caused by lumbar disc herniation or other pathologies of the lumbar discs or vertebrae and presents as pain radiating from the back into the leg, usually in a dermatomal pattern corresponding to the compressed nerve root. The majority of patients with sciatic pain improve over time with conservative treatment ${ }^{1,2}$. There is, however, a subgroup of patients who fail to respond to such conservative treatment. Periradicular application of local anesthetic with costicosteroids $^{3-7}$ and periradicular application of ozone $\mathrm{e}^{9-11}$ has previously been shown to be efficient in this group of patients. During the compression of the nerve root due to disc herniation, spinal stenosis, spondylolisthesis and other causes, there is a strong inflammatory reaction and therefore, periradicular infiltration of corticosteroids would seem like a reasonable treatment option ${ }^{12-15}$. Other common causes of sciatic pain with radicular irritation are lumbar foraminal stenosis, spondylolisthesis and postoperative radicular fibrosis causing compression or irritation of the nerve root. There is only a little evidence that compares the outcomes of periradicular infiltration in patients with the above-mentioned pathology with those monitored for herniated discs. The purpose of this study was to investigate the effect of different corticosteroids and ozone in periradicular application, to compare treat- 
ment agents, and to asses whether therapy has any benefit in specific subgroups of patients with radicular pain.

\section{MATERIAL AND METHODS}

The patients were enrolled from January 2019 to April 2020. One hundred and fifty patients with radicular leg pain and MRI-confirmed nerve root compression due to lumbar disc herniation, foraminal stenosis, lumbar spinal stenosis, spondylolisthesis and postoperative periradicular fibrosis were divided into three groups. Conservative treatment had failed in all the patients; we excluded those patients with allergies to treatment agents, inability to complete the questionnaire, pregnancy or Cauda equina syndrome. Upon the trial enrollment, an information sheet describing the study was given to the patients and informed consent was obtained from all the patients prior to trial entry. The patients then completed a standard visual analogue score (VAS) for leg pain prior to the PRT therapy and 12 weeks after the PRT therapy. There were no complications during epidural steroid application.

\section{Periradicular treatment procedure}

The patients were enrolled into three groups to receive either $1 \mathrm{~mL}$ of betamethasone (Diprophos) +4 $\mathrm{mL} 0.25 \%$ bupivacaine, $1 \mathrm{~mL}$ of methylprednisolone (Depomedrone) $+4 \mathrm{~mL} 0.25 \%$ bupivacaine or $10 \mathrm{~mL}$ ozone generated by a TAO 80 ozone generator. The periradicular application was performed using CT control image in three repeated applications after 7 days. All the applications were performed by a senior neurosurgeon (DK). The patients were prone on the CT scanning table and a spinal needle was used to approach the nerve root under the CT image guidance. Once a satisfactory position of the needle had been confirmed, the treatment agent + iodine contrast medium or $10 \mathrm{~mL}$ of ozone was injected slowly around the nerve root and a control CT scan was obtained to monitor the application.

\section{Statistical analysis}

Statistical analysis was performed using SPSS Statistics for Windows v22.0 (IBM Corp., Armonk, NY, USA) and SciPy v1.5.2 (ref Nature Methods, 2020), Seaborn v0.11.0 and scikit-posthocs v0.6.5 Python libraries.

The normality of all the data was tested using the Shapiro-Wilk test. An age comparison of the groups was

performed using the nonparametric Kruskal-Wallis H-test. The between-group difference in sex and indication was tested using the Fisher exact test.

The within-group treatment effect (change of leg pain VAS prior to and after therapy) was tested using a paired Wilcoxon signed-rank test. The between-group difference in treatment effect (VAS prior to therapy minus VAS after therapy) was tested using the Kruskal-Wallis H-test and Dunn's post-hoc tests.

The dependency between the treatment effect and the indication for each group was tested using the KruskalWallis H-test and Dunn's post-hoc tests.

\section{RESULTS}

The Shapiro-Wilk normality test of age for individual groups showed that the age for the Depomedrone and ozone patients comes from normal distribution ( $P=0.532$ and $P=0.356$ ) but the age of Diprohos patients does not $(P=0.029)$. However, the subsequent Kruskal-Wallis H-test did not reveal any significant age difference between the groups $(P=0.374)$; see Table 1 .

No significant difference in sex and indication was found between the groups $(P=0.605$ and $P=0.637$, respectively); see Table 2.

The Wilcoxon signed-rank test of in-group treatment effect (leg pain VAS prior to and after therapy) showed a statistically significant $(P<0.001)$ difference for all three groups. The median decrease of VAS was 4.0, 3.0, and 2.0 for Diprophos, Depomedrone and ozone, respectively; see Fig. 1.

The between-group testing of the treatment effect (VAS prior to therapy minus VAS after therapy) using the Kruskal-Wallis H-test revealed a statistically significant difference between the groups $(P<0.001)$. Dunn's posthoc tests showed a statistically significant difference in treatment effect between the groups of Diprophos and

Table 1. Age characteristics of individual groups.

\begin{tabular}{lcccc}
\hline Age & Diprophos & Depomedrone Ozone & $P$ \\
\hline Mean & 51.4 & 50.0 & 54.2 & \\
STD & 16.4 & 14.0 & 14.7 & \\
Median & 47.5 & 48.0 & 56.5 & 0.374 \\
Minimum & 25.0 & 21.0 & 24.0 & \\
Maximum & 83.0 & 81.0 & 84.0 & \\
\hline
\end{tabular}

Table 2. Sex and indication characteristics of the individual groups.

\begin{tabular}{|c|c|c|c|c|c|c|c|c|}
\hline & & \multirow{2}{*}{\multicolumn{2}{|c|}{$\begin{array}{c}\text { Diprophos } \\
\text { Count \% }\end{array}$}} & \multicolumn{2}{|c|}{ Depomedrone } & \multicolumn{2}{|c|}{ Ozone } & \multirow[t]{2}{*}{$P$} \\
\hline & & & & Count & $\%$ & Count & $\%$ & \\
\hline \multirow[t]{2}{*}{ Sex } & Male & 25 & 50.0 & 26 & 52.0 & 23 & 46.0 & \multirow[t]{2}{*}{0.605} \\
\hline & Female & 25 & 50.0 & 24 & 48.0 & 27 & 54.0 & \\
\hline \multirow[t]{5}{*}{ Indication } & Foraminal stenosis & 10 & 20.0 & 8 & 16.0 & 5 & 10.0 & \multirow{5}{*}{0.637} \\
\hline & Disc herniation & 10 & 20.0 & 15 & 30.0 & 10 & 20.0 & \\
\hline & Spinal stenosis & 13 & 26.0 & 14 & 28.0 & 18 & 36.0 & \\
\hline & Postoperative fibrosis & 7 & 14.0 & 5 & 10.0 & 10 & 20.0 & \\
\hline & Spondylolisthesis & 10 & 20.0 & 8 & 16.0 & 7 & 14.0 & \\
\hline
\end{tabular}


Table 3. Treatment effect (VAS change prior to and after therapy) for individual drugs and indications.

\begin{tabular}{|c|c|c|c|c|c|c|c|}
\hline & Treatment effect & Spinal stenosis & Spondylolisthesis & Disc herniation & $\begin{array}{c}\text { Postoperative } \\
\text { stenosis }\end{array}$ & $\begin{array}{c}\text { Foraminal } \\
\text { fibrosis }\end{array}$ & $P$ \\
\hline \multirow{5}{*}{ Diprophos } & Mean & 4.4 & 2.4 & 5.4 & 3.0 & 4.5 & \multirow{5}{*}{0.015} \\
\hline & STD & 2.1 & 1.8 & 1.6 & 2.0 & 1.7 & \\
\hline & Median & 4.0 & 2.0 & 5.0 & 3.0 & 4.5 & \\
\hline & Minimum & 1.0 & 0.0 & 3.0 & 0.0 & 1.0 & \\
\hline & Maximum & 8.0 & 6.0 & 8.0 & 6.0 & 7.0 & \\
\hline \multirow{5}{*}{ Depomedrone } & Mean & 2.7 & 1.5 & 4.0 & 2.6 & 3.1 & \multirow{5}{*}{0.164} \\
\hline & STD & 2.2 & 2.1 & 2.4 & 2.4 & 1.1 & \\
\hline & Median & 2.5 & 0.5 & 4.0 & 4.0 & 3.5 & \\
\hline & Minimum & 0.0 & 0.0 & 0.0 & 0.0 & 1.0 & \\
\hline & Maximum & 6.0 & 6.0 & 10.0 & 5.0 & 4.0 & \\
\hline \multirow{5}{*}{ Ozone } & Mean & 2.9 & 0.7 & 3.1 & 2.6 & 1.2 & \multirow{5}{*}{0.005} \\
\hline & STD & 1.1 & 1.1 & 1.4 & 1.6 & 1.6 & \\
\hline & Median & 3.0 & 0.0 & 3.5 & 3.0 & 1.0 & \\
\hline & Minimum & 1.0 & 0.0 & 0.0 & 0.0 & 0.0 & \\
\hline & Maximum & 5.0 & 3.0 & 5.0 & 5.0 & 4.0 & \\
\hline
\end{tabular}

Depomedrone patients $(P=0.019)$ and between the groups of Diprophos and ozone patients $(P<0.001)$; see Fig. 2.

The Kruskal-Wallis testing of the dependency between the treatment effect and indication for each group showed statistically significant dependency for the Diprophos group $(P=0.015)$ and the ozone group $(P=0.005)$; see Table 3. Dunn's post-hoc tests then revealed a statistically significant difference between the spondylolisthesis and disc herniation ( $P=0.019$ ) indications for the Diprophos group and between the spinal stenosis and spondylolisthesis $(P=0.022)$ and spondylolisthesis and disc herniation indications $(P=0.016)$ for the ozone group; see Fig. 3.

\section{DISCUSSION}

Periradicular application of corticosteroids and ozone has been widely used for the treatment of radicular pain in several pathologies. Radicular pain is thought to be induced by ectopic firing or excessive excitation from a nerve root and various chemical mediators can induce ectopic firing ${ }^{16,17}$. Corticosteroids can affect several mechanisms leading to radicular pain such as the inhibition of PGE2, stabilization of the neural membrane, anti-inflammatory response, and anti-edematous response, and they can facilitate the recovery of nerve conduction velocity ${ }^{18-20}$. The effect of ozone therapy is thought to be because of the decrease in the irritation of the sinuvertebral nerve ends and because of the antinociceptive action. These actions are due to the decreased reaction of following factors $^{21,22}$ :

- the discirculatory factor as decrease in the edema of the surrounding tissues

- the dislocation factor due to partial restoration of the amortization properties of the affected disc; as a result, improvement of its metabolism and the activation of trophic influences occur.

- the aseptic-inflammatory factor induced by the synthesis of prostaglandins as inflammation mediators and

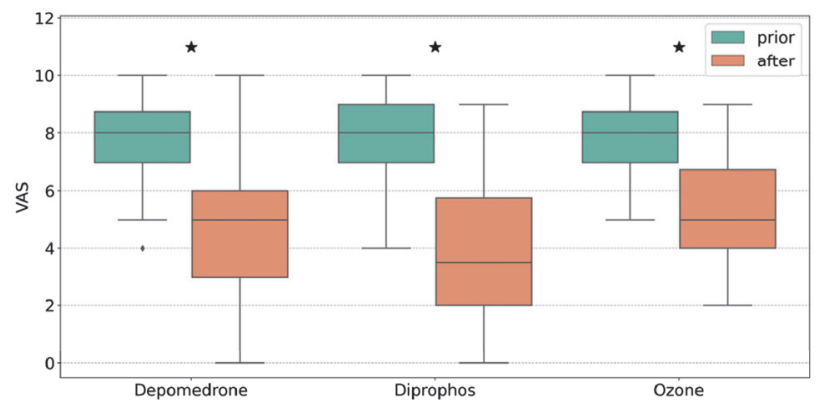

Fig. 1. Grouped boxplots showing the distribution of VAS for individual drugs prior to and after therapy. The asterisks denote a statistically significant $(P<0.001)$ difference between VAS prior to and after therapy for each group.

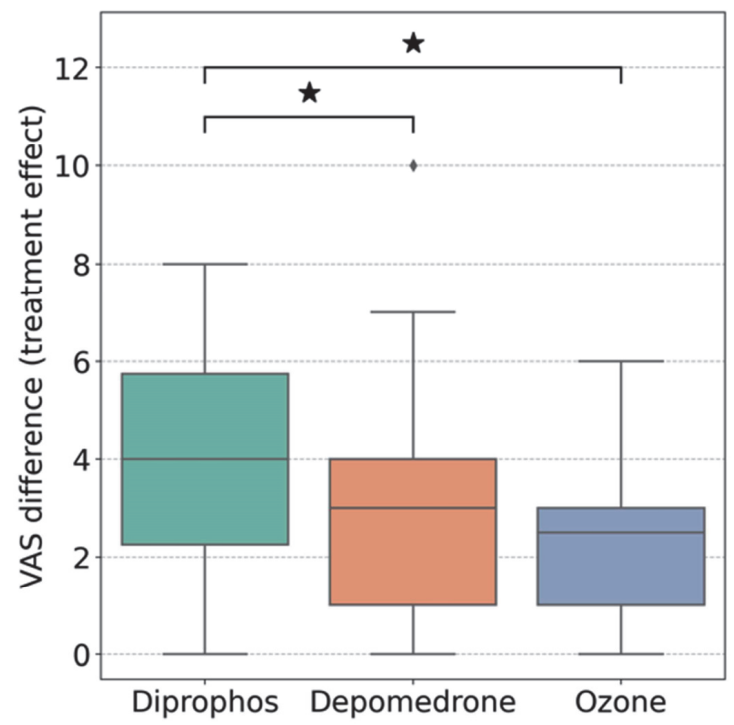

Fig. 2. Boxplots visualizing between-group difference in treatment effect. The asterisks denote a statistically significant difference between the Diprophos and Depomedrone patients $(P=0.019)$ and between the groups of Diprophos and ozone patients $(P<0.001)$. 

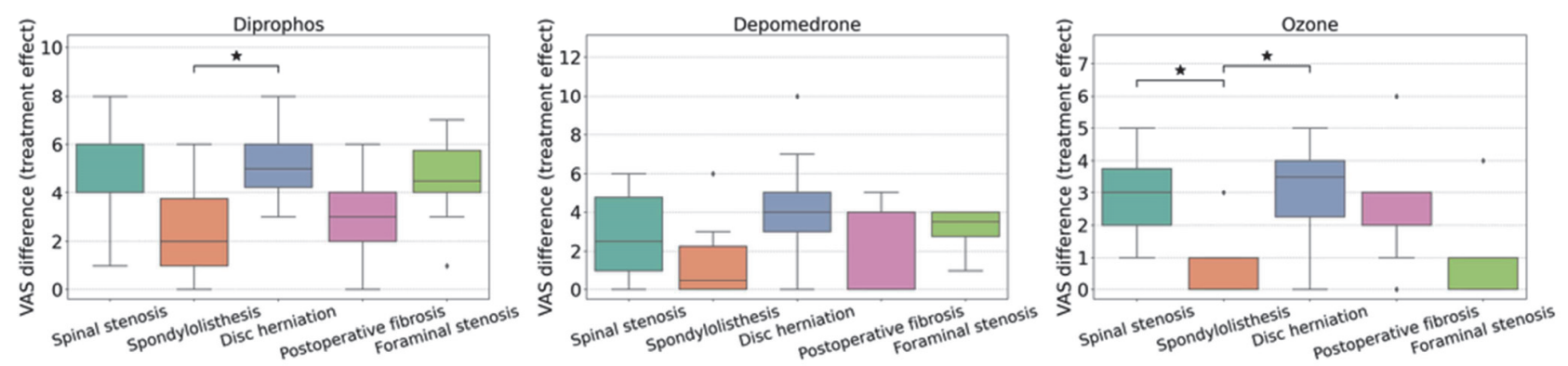

Fig. 3. Treatment effect (VAS change prior to and after therapy) for individual drugs and indications. The asterisks denote statistically significant differences between the given indications.

the activation of cellular immunity contributing to the fast elimination of "foreign" components. As a result, it comes to a significant decrease in afferent flow from the periphery to the CNS.

- direct oxidation of algo peptides

- decrease in under-oxidated products in spasmodic muscles

- an increase in the excitability threshold of pain receptor membranes (membrane-stabilizing effect).

A single injection of periradicular application has only short effect, but multiple injections have produced long-term results ${ }^{7-23}$. Diprophos consists of two agents betamethasone natrii phosphas and betamethasone dipropionas. The first part is a fast-acting form of $2 \mathrm{mg}$ of betamethasone and the second is a long-lasting depot consisting of $5 \mathrm{mg}$ of betamethasone. Depomedrone consists of $40 \mathrm{mg}$ of methylprednisolone.

Our study showed a statistically significant treatment effect $(P<0.001)$ in all three groups. The statistically significant effect was higher in betamethasone (Diprophos) versus methylprednisolone (Depomedrone) $(P=0.019)$ and Diprophos versus ozone $(P<0.001)$. Diprophos also showed the highest decrease of VAS after therapy versus VAS prior to therapy (median decrease $=4$ ) compared to Depomedrone and ozone (median decrease $=3$ and 2, respectively). The explanation of the results suggests that the second, depot part of the Diprophos may be responsible for the higher effect of the PRT therapy. Subgroup analysis was performed to examine if there was any difference in the outcome between the patients who had radicular pain secondary to lumbar disc herniation or to the other four indication groups. The statistically significant outcome was better with the indication of disc herniation and the spondylolisthesis indication $(P=0.019)$ for the Diprophos group and between spinal stenosis and spondylolisthesis $(P=0.022)$ and spondylolisthesis and disc herniation $(P=0.016)$ for the ozone group.

$\mathrm{Ng}$ et al. ${ }^{4}$ published their study to compare the results of corticosteroids versus bupivacaine only, displaying no significant difference between the groups for chronic radicular pain. Our results of the study show a statistical difference between the groups, and Diprophos seems to be the best agent for the application. Recent publications by Smith $^{24}$ published comprehensive review of transforaminal application of corticosteroids in lumbar spine. Their results proved, equally to our study, strong evidence that lumbar transforaminal injection of steroids is an effective treatment for radicular pain due to disc herniation. There is a lack of high-quality evidence demonstrating their effectiveness for the treatment of radicular pain due to spinal stenosis, though small studies suggest a possible benefit. Cohen ${ }^{25}$ focused his publication at the risk of complication during epidural steroid application. Their results showed that the risk of complications for transforaminal epidural steroid application is greater with particulate corticosteroids. Nonparticulate corticosteroids, which are often recommended as first-line therapy, may have a short duration of effect but the risk of complication is lower. In our study we had no complication during epidural steroid application despite the fact we used particulate steroids. Ozcan ${ }^{26}$ in his study, proved the efficacy of paravertebral Ozone application for low back pain.

\section{CONCLUSION}

Periradicular infiltration is a safe and simple procedure that can be performed to provide radicular pain relief. Clinical improvement occurred in all three groups, but Diprophos showed the statistically best treatment effect compared to Depomedrone and ozone. Disc herniation resulting for radicular pain had a statistically significant better effect in comparison with spondylolisthesis in the Diprophos and ozone groups, but the ozone group showed heterogeneity depending on treatment effect and indication.

Author contributions: DK: manuscript writing, data analysis; DK,LH,MV: final approval; JV,JZ: statistical evaluation; JJ,DP: data analysis, literature search.

Conflict of interest statement: No funds were received in support of this work. No benefits in any form have been or will be received from a commercial party related directly or indirectly to the subject of this manuscript. The drugs are approved by the corresponding national agency for this indication. All the patients signed informed consent forms. 


\section{REFERENCES}

1. Weber $\mathrm{H}$. The natural history of disc herniation and the influence of inter- vention. Spine 1994;19:2234-8.

2. Weber $\mathrm{H}$, Holme I, Amlie $\mathrm{E}$. The natural cause of acute sciatica with nerve root symptoms in a double-blind placebo-controlled tria evaluating the ef- fect of piroxicam. Spine 1993;18:1433-8.

3. Tafazal S, Ng L,j Chaudhary N, Sell P. Corticosteroids in peri-radicular infiltration for radicular pain: a randomised double blind controlled trial. One year results and subgroup analysis. Eur Spine 2009;18:1220-25.

4. Ng L, Tafazal S, Chaudhary N, Sell P. The Efficacy of Corticosteroids in Periradicular Infiltration for Chronic Radicular Pain -A Randomized Double-Blind, Controlled Trial. Soine 2005;30(8):857-62.

5. Koes BW, Scholten R, Mens J. Efficacy of epidural injections for low back pain and sciatica: a systemic review of randomised clinical trials. Pain1995;63:279-88.

6. Karppinen J, Malmivaara A, Kurunlahti M. Peri-radicular infiltration for sciatica: a randomised controlled trial. Spine 2001;13:1056-67.

7. Lutz GE, Vad VB, Wisneski RJ. Fluoroscopic transforaminal lumbar epidural steroids: an outcome study. Arch Phys Med Rehabi 1998:79:1362-6.

8. Weiner BK, Fraser RD. Foraminal injection for lateral lumbar disc herniation. J Bone Joint Surg Br 1997;79B:804-7.

9. De Oliveira Magalhaes F, Dotta L, Sasse A. Ozone therapy as a treatment for low back pain secondary to herniated disc: a systematic review and meta-analysis of randomized controlled trials. Pain Physician 2012;15:E115-129.

10. Magalhaes FN, Dotta L, Sasse A. Ozone therapy as a treatment for low back pain secondary to herniated disc: a systematic review and meta-analysis of randomized controlled trials. Pain Physician 2012;15(2):E115-129.

11. Murphy K, Muto M, Steppan J. Treatment of contained herniated lumbar discs with ozone and corticosteroids: A pilot clinical study. Can Assoc Radiol J 2015;66:337-84.

12. Marshall LL, Trethewie ER, Curtain CC. Chemical radiculitis, a clinical physiological and immunological study. Clin Orthop 1977;129:61-7.

13. Olmarker K, Blomquist J, Stromberg J. Inflammatogenic properties of nucleus pulposus. Spine 1995;20:665-9.
14. Olmarker K, Rydevik B, Nordborg C. Autologous nucleus pulposus induces neurophysiologic and histologic changes in procaine cauda equina nerve roots. Spine 1993;18:1425-32.

15. Olmarker K, Byrod G, Cornefjord M. Effects of methylprednisolone on nucleus pulposusinduced nerve root injury. Spine 1994;19:1803-8.

16. Atsuta $Y$, Sugawara $O$, Muramoto $T$. In vitro analysis of ectopic firing generated in the lumbar nerve root. J Jpn Spine Res Soc 1993;4:S74.

17. Atsusta $Y$, Iwahara T, Sugawara O. The study of generating and suppressive factors of ectopic firing in the lumbar dorsal root using an in vitro model. Rinsho Seikei Geka 1994;29:441-8.

18. Devor M, Govrin-Lippmann R, Raber P. Corticosteroids suppress ectopic neural discharge originating in experimental neuromas. Pain 1985;22:127-37.

19. Hong CZ, Cheng BB, Liu AY. Local steroid injection: its effect on recovery of nerve conduction in experimental neuropathy. Arch Phys MedRehabil 1990;71:42-5.

20. Berman AT, Garbarino JL, Fisher SM. The effects of epidural injection of local anaesthetics and corticosteroids on patients with lumbosciatic pain.Clin Orthop 1991;188:144.

21. Wentworth P. Evidence for antibody catalyzed ozone formation in bacterial killing and inflammation. Science 2002;298:2195-99.

22. Pellicanò F, Martinetti F, Tavanti V. The Italian Oxygen-Ozone Therapy Federation (FIO) study on oxygen-ozone treatment of herniated disc. Int J Ozone Ther 2007;6:7-15.

23. Riew KD, Yin Y, Gilula L. The effect of nerve rooti njections on the need for operative treatment of lumbar radicular pain. J Bone Joint Surg Am 2000;82A:1589-93.

24. Smith CC, McCormick Z, Mattie R, MacVicar J, Duszynski B, Stojanovic MP. The Effectiveness of Lumbar Transforaminal Injection of Steroid for the Treatment of Radicular Pain: A Comprehensive Review of the Published Data. Pain Med 2020;21(3):472-87. doi: 10.1093/pm/ pnz160

25. Cohen PS, Greuber E, Vought K, Lisssin D. Safety of Epidural Steroid Injections for Lumbosacral Radicular Pain: Unmet Medical Need. Clin J Pain 2021;37(9):707-17.

26. Ozcan C, Polat O, Celik H, Ucar BY. The Effect of Paravertebra Ozone Injection in the Treatment of Low Back Pain. Pain Pract 2019;19(8):821-25. doi: 10.1111/papr.12812 